Pacific Journal of Mathematics

ALEXANDER DUALITY

Wilda H. JULIAN, RaY Mines, HI AND Fred Richman 


\section{ALEXANDER DUALITY}

\section{William Julian, Ray Mines and Fred Richman}

The Alexander duality theorem is developed in a manner that is constructive in the sense of Bishop, yielding a constructive JordanBrouwer theorem.

0. Introduction. The Alexander duality theorem, which generalizes the Jordan curve theorem and the Jordan-Brouwer theorem, relates the structure of a compact subset of a $d$-dimensional sphere to that of its complement. The structure of the complement is not completely determined by the structure of the subset. Indeed, the homotopy class of the complement is not determined by the homeomorphism class of the set, as the Alexander horned sphere illustrates. Nevertheless certain connectivity properties of the complement are so determined, such as the number of connected components. This consequence of duality generalizes the Jordan-Brouwer theorem that the complement in the $d$-sphere of a homeomorph of the $(d-1)$-sphere has two components.

We develop the duality theorem in a manner that is constructive in the sense of Bishop [3], thus generalizing the work in [1] on the constructive Jordan curve theorem to obtain a constructive Jordan-Brouwer theorem. The constructive approach requires showing how to use the information given by a homeomorphism of the $(d-1)$-sphere with a subset $T$ of the $d$-sphere $S^{d}$, to construct two points in $S^{d}$ of positive distance away from $T$ such that any path joining them comes arbitrarily close to $T$; and, given a point in $S^{d}$ of positive distance from $T$, how to construct a path joining it to one of the two points by a polygonal path that is bounded away from $T$. The strategy, as in the classical situation, is to associate a homeomorphism invariant group $H^{d-1}(X)$ to each compact space $X$ so that if $T$ is a compact subset of $S^{d}$ and $H^{d-1}(T)=H^{d-1}\left(S^{d-1}\right)$, then $T$ satisfies the conclusion of the Jordan-Brouwer theorem. To this end we develop a constructive Čech cohomology theory for compact spaces.

In [6] a constructive Vietoris homology theory is developed for compact spaces, in which the homology objects are inverse systems of (finitely presented) abelian groups. These more general objects made it possible to get a theory that is both exact and continuous, an impossibility if the objects are required to be abelian groups [4; X.4.1]. Moreover some information is lost if we pass to the inverse limit: The first homology group of a circle with a missing piece is trivial, but this fact provides no information as to the size of the missing piece. However if we are given 
that the associated inverse system of groups is trivial, then we can calculate the size of the missing piece. It is essential that we know this size if we are to prove that the complement in the plane of such a punctured circle is pathwise connected.

The situation for cohomology is different, as the category of direct systems of finitely generated abelian groups is isomorphic to the category of abelian groups. If we are given that the cohomology group of the punctured circle is trivial, then we can compute the size of the puncture. From a constructive point of view, the cohomology objects are countably presented abelian groups, that is, groups generated by a countable number of generators subject to a countable number of relations. The theory of these groups is developed in $§ 1$.

We develop in $\$ 2$ a homeomorphism invariant Čech cohomology theory that satisfies the Eilenberg-Steenrod axioms. In $\S 3$ we show that the cohomology of an inverse limit of compact spaces is the direct limit of the cohomology groups of the spaces. This enables us to compute the cohomology group of a compact subset $T$ of a sphere as the direct limit of the cohomology groups of approximating polyhedra. By suitably choosing the approximating polyhedra, we can construct isomorphisms between their cohomology groups and the homology groups of polyhedra approximating the complement of $T$ via the combinatorial duality theorem. There are no peculiarly constructive problems with this theorem as it deals entirely with finite objects and operations. However we do have to observe that the isomorphisms form a natural equivalence of functors in the appropriate category. This is done in $\$ 5$. In $\S 6$ we prove the JordanBrouwer theorem.

1. Countably presented groups. An abelian group is countably presented if it can be written as a quotient of a countable free group by a countable subgroup. From a classical point of view these groups are simply countable groups; constructively they carry a little more information. For example if we let $H$ be the subgroup of the additive group $Z$ of integers which is $Z$ or 0 depending on the truth of some proposition, then $Z / H$ is a countable group that cannot necessarily be asserted to be countably presented. The two notions coincide for discrete groups, that is, for groups in which the alternative " $x=y$ or $x \neq y$ " is decidable. Countably presented groups arise in this paper as cohomology groups of compact spaces, and these groups need not be discrete.

An abelian group is finitely presented if it can be written as the quotient of a finite rank free group by a finitely generated subgroup. Classically any finitely generated abelian group is finitely presented. It is easily seen that countably presented groups are precisely the countable direct limits of finitely presented groups. More is true: the category of 
countably presented groups is isomorphic to the category of countable direct systems of finitely presented groups. This latter category is a standard construction which we shall briefly outline, assuming the reader to be familiar with direct and inverse limits.

The direct limit $\lim _{\rightarrow} H_{\alpha}$ of a direct system $\left(H_{\alpha}\right)_{\alpha \in A}$ is the union of the $H_{\alpha}$, two elements being equal if their images are equal in some $H_{\beta}$. The inverse limit $\lim _{\leftarrow} K_{\alpha}$ of an inverse system $\left\{K_{\alpha}\right\}_{\alpha \in A}$ consists of those elements of the product $\Pi K_{\alpha}$ whose coordinates respect the maps in the system.

Definition. Let $C$ be a category. The category $\operatorname{Dir}(C)$ has as its objects the direct systems $\left\{H_{\alpha}\right\}_{\alpha \in A}$ of objects in $C$. A map between direct systems $\left\{H_{\alpha}\right\}_{\alpha \in A}$ and $\left\{K_{\beta}\right\}_{\beta \in B}$ is an element of $\lim _{\leftarrow} \lim _{\beta} \operatorname{Hom}\left(H_{\alpha}, K_{\beta}\right)$.

THEOREM 1.1. The direct limit functor is an isomorphism between the category of countable direct systems of finitely presented groups, and the category of countably presented groups.

Proof. Let $A_{0} \rightarrow A_{1} \rightarrow \cdots$ and $B_{0} \rightarrow B_{1} \rightarrow \cdots$ be direct systems of finitely presented groups. Let $A_{\infty}$ and $B_{\infty}$ be their direct limits. We must show that any map $A_{\infty} \rightarrow B_{\infty}$ is induced by a map between the direct systems, and that any map between the systems that induces the zero map on $A_{\infty}$ is zero. We construct a map inducing $f$ as follows. Given $i$, let $S$ be a finite set of generators for $A_{i}$ and $R$ a finite set of generators for the relations on $S$ in $A_{i}$. Then $f(S)$ can be regarded as a subset of some $B_{j}$ and, since $f(R)$ hold in $B_{\infty}$, we can choose $j$ so that $f(R)$ hold in $B_{j}$. This gives a map $A_{i} \rightarrow B_{j}$. The maps arising in this manner define a map between the two direct systems that induces $f$ on $A_{\infty}$.

As each $A_{i}$ is finitely generated, any map between the two systems that induces the zero map on $A_{\infty}$ is zero.

THEOREM 1.2. Let $G_{0} \rightarrow G_{1} \rightarrow \cdots$ be a direct system of finitely presented groups. If the direct limit of this system is finitely presented, then we can reindex the system so that $G_{i}=A_{i} \oplus M_{i}$ where $M_{i}$ goes to zero in $G_{i+1}$ and $A_{i}$ is mapped isomorphically onto $A_{i+1}$.

Proof. We may reindex so that each $G_{i}$ contains a finite set of generators of $G_{\infty}$ and that the relations holding on this set in $G_{\infty}$ also hold in $G_{i}$. These generators generate the subgroup $A_{i}$ of $G_{i}$, which maps isomorphically onto $A_{i+1}$. Each of the finite number of generators of $G_{i}$ is equal in $G_{\infty}$ to an element of $A_{i}$. Hence we may reindex so that $G_{i}$ maps into $A_{i+1}$. The kernel of the map from $G_{i}$ to $G_{i+1}$ is $M_{i}$. 
2. Čech cohomology of totally bounded pairs. A subspace $Y$ of a metric space $S$ is $\varepsilon$-dense if given a point $x$ in $X$ we can find a point $y$ in $Y$ such that $d(x, y)<\varepsilon$. The subspace $Y$ is dense if it is $\varepsilon$-dense for every $\varepsilon>0$. The space $X$ is totally bounded if for each $\varepsilon>0$ it has a finite $\varepsilon$-dense subspace. We consider the empty set to be finite, hence totally bounded. A totally bounded pair consists of a totally bounded space $X$ and a totally bounded subspace $X^{\prime}$. We shall sometimes denote such a pair simply by $X$. For $x$ in $X$ we let $N_{\delta}(x)$ denote the set of all points $y$ in $X$ such that $d(x, y)<\delta$. A cover of a totally bounded pair $X$ consists of a finite family $I$ of nonempty subsets of $X$ and positive numbers $\varepsilon \geq \delta$ such that

(a) If $x$ and $y$ are in $U \in I$, then $d(x, y)<\varepsilon$.

(b) If $x \in \mathrm{X}$, then there is $U$ in $I$ such that $N_{\delta}(x) \subset U$.

We shall denote the cover simply by $I$ and write $\varepsilon(I)$ and $\delta(I)$ for $\varepsilon$ and $\delta$ to indicate to which cover they apply. Let $I^{\prime}$ denote the subfamily of $I$ consisting of those subsets containing points of $X^{\prime}$. We now show that covers with pleasant constructive properties exist in abundance.

THEOREM 2.1. Let $X$ be a totally bounded pair and $e>0$. Then there exists a cover I of $X$ for which $\varepsilon(I)<$ e satisfying

(a) Every set in I is open and totally bounded.

(b) Every finite subfamily $F$ of I either has empty or nonempty intersection.

(c) Properties (a) and (b) hold for the family $\left(U \cap X^{\prime}: U \in I\right)$ of subsets of $X^{\prime}$.

(d) The subfamily $I^{\prime}$ of I consisting of those subsets containing points of $X^{\prime}$ is finite.

Proof. Let $Y$ be a finite $e / 4$-dense subset of $X$. For each finite subset $Z$ of $Y$ let $f_{Z}(x)=\sup \{d(x, z): z \in Z\}$. By [2; Theorem 0.1] we can choose $\theta$ in $(e / 4, e / 2)$ so that $\left\{x \in X: f_{Z}(x)<\theta\right\}$ and $\left\{x \in X^{\prime}: f_{Z}(x)\right.$ $<\theta\}$ are totally bounded for each finite subset $Z$ of $Y$. If $I=\left\{N_{\theta}(y): y\right.$ $\in Y\}$, then (a), (b) and (c) hold. Let $\varepsilon(I)=2 \theta<e$ and $\delta(I)=\theta-e / 4$. Then $N_{\theta}(y)$ is nonempty, and $N_{\delta(I)}(x) \subset N_{\theta}(y)$, where $y$ is an element of the $e / 4$-approximation $Y$ such that $d(x, y)<e / 4$. Part (d) follows from the total boundedness of the sets $U \cap X^{\prime}$.

Each cover $I$ gives rise to simplicial pair, which we shall also denote by $I$, by declaring a finite subset of $I\left(I^{\prime}\right)$ to be a simplex if its intersection contains a point of $X\left(X^{\prime}\right)$. A simplicial map is a vertex map on simplicial pairs that takes simplices to simplices. Two simplicial maps $\alpha$ and $\beta$ are contiguous if $\alpha \sigma \cup \beta \sigma$ is a simplex for all simplices $\sigma$. We say that $\alpha$ and $\beta$ are combinatorially homotopic, and write $\alpha \sim \beta$, if there exist simplicial maps $\alpha=\alpha_{0}, \alpha_{1}, \ldots, \alpha_{m}=\beta$ such that $\alpha_{i}$ is contiguous to $\alpha_{i+1}$ for 
$0 \leq i<m$. Combinatorially homotopic maps induce the same maps on homology and cohomology.

Let $f: X \rightarrow Y$ be a map of totally bounded pairs, that is, $f$ is uniformly continuous and $f\left(X^{\prime}\right) \subset Y^{\prime}$. Let $I$ and $J$ be covers of $X$ and $Y$ respectively. Then we say that $f_{J}^{I}$ is defined if there is a simplicial map $\alpha: I \rightarrow J$ such that $f(U) \subset \alpha(U)$ for all $U$ in $I$. If $\beta$ is also a map from $I$ to $J$ such that $f(U) \subset \beta(U)$ for all $U$ in $I$, then $\alpha$ and $\beta$ are contiguous. Thus setting $f_{J}^{I}(U)=\alpha(U)$ uniquely defines a simplicial map up to combinatorial homotopy.

If $f$ and $g$ map $X$ to $Y$, and $d\left(f x_{1}, g x_{2}\right)<\delta(J)$ whenever $d\left(x_{1}, x_{2}\right)<$ $\varepsilon(I)$, then $f_{J}^{I}$ and $g_{J}^{I}$ are defined and $f_{J}^{I} \sim g_{J}^{I}$.

THEOREM 2.2. Let $f: X \rightarrow Y$ and $g: Y \rightarrow Z$ be maps of totally bounded pairs. Let $I, J$ and $K$ be covers of $X, Y$ and $Z$ respectively. If $f_{J}^{I}$ and $g_{K}^{J}$ are defined, then $(g f)_{K}^{I}$ is defined and $(g f)_{K}^{I} \sim g_{K}^{J} f_{J}^{I}$.

We say that a cover $I$ is a refinement of a cover $J$ of the same pair $\left(X, X^{\prime}\right)$ if $\operatorname{id}_{J}^{I}$ is defined. The covers of Theorem 2.1 form a cofinal directed set under this relation since $\mathrm{id}_{J}^{I}$ is defined if $\varepsilon(I)<\delta(J)$. The cohomology group $H^{n}\left(X, X^{\prime}\right)$ is defined to be the direct limit of the groups $H^{n}\left(I, I^{\prime}\right)$ over the covers $I$ of $\left(X, X^{\prime}\right)$. Hence, by Theorems 1.1 and 2.1, $H^{n}\left(X, X^{\prime}\right)$ is countably presented.

The groups $H^{n}$ do not distinguish a space from its completion:

THEOREM 2.3. Let $\left(X, X^{\prime}\right) \subset\left(Y, Y^{\prime}\right)$ be an inclusion of totally bounded pairs. If $X$ is dense in $Y$ and $X^{\prime}$ is dense in $Y^{\prime}$, then $H^{n}\left(X, X^{\prime}\right)=H^{n}\left(Y, Y^{\prime}\right)$.

Proof. If $I$ is a cover of $X$ as in Theorem 2.1, then $J=(U \cap Y: U \in I)$ is a cover of $Y$ with the same simplicial structure as $I$.

We now show that $H^{n}$ provides a cohomology theory in the sense of Eilenberg-Steenrod. That $H^{n}$ is a functor, that the coboundary map is natural, that $H^{n}=0$ on one point spaces for $n \neq 0$, and that the cohomology sequence

$$
H^{n^{\prime}-1}\left(X^{\prime}\right) \rightarrow H^{n}\left(X, X^{\prime}\right) \rightarrow H^{n}(X) \rightarrow H^{n}\left(X^{\prime}\right) \rightarrow H^{n+1}\left(X, X^{\prime}\right)
$$

is exact, is immediate from the corresponding facts for $H^{n}(I)$. We need to verify the excision and homotopy axioms.

THEOREM 2.4. Let $\left(X, X^{\prime}\right)$ be a totally bounded pair and $Y$ a totally bounded subspace of $X$ such that $Y^{\prime}=Y \cap X^{\prime}$ is totally bounded. Suppose there is $\delta>0$ such that for each $x$ in $X$ either $x \in Y$ or every point within $\delta$ of $x$ is in $X^{\prime}$. Then the homomorphism $H^{n}\left(X, X^{\prime}\right) \rightarrow H^{n}\left(Y, Y^{\prime}\right)$ induced by the inclusion $\left(Y, Y^{\prime}\right) \subset\left(X, X^{\prime}\right)$ is an isomorphism. 
Proof. If $I$ is a cover of $X$, and $\varepsilon(I)<\delta$, then there is a finite subfamily $I_{0}$ of $I$, every element of which intersects $Y$, such that every simplex of $I$ is either contained in $I_{0}$ or contained in $I^{\prime}$. Indeed choose $I_{0}$ so that if $U$ is in $I_{0}$ then $U \cap Y$ is nonempty, and if $U$ is not in $I_{0}$ then $U \subset X^{\prime}$. If a simplex of $I$ has some vertex not in $I_{0}$, then all vertices are in $I^{\prime}$. The natural map $H^{n}(I) \rightarrow H^{n}\left(I_{0}\right)$ is an isomorphism.

If $J$ is a cover of $Y$, and $I$ is a cover of $X$ such that $\varepsilon(I)<$ $\min (\delta, \delta(J) / 2)$, let $\alpha: I_{0} \rightarrow J$ be such that $\alpha U$ contains a $\delta(J)$ neighborhood in $Y$ of a point in $U \cap Y$. Then $\alpha^{*}: H^{n}(J) \rightarrow H^{n}\left(I_{0}\right) \cong H^{n}(I)$ gives the desired inverse map.

THEOREM 2.5. Let $X$ be a totally bounded pair and $T$ the unit interval. Let $f_{t}: X \rightarrow X \times T$ be defined by $f_{t}(x)=(x, t)$. Then $f_{0}$ and $f_{1}$ induce the same map on cohomology.

Proof. We may assume that the metric on $X \times T$ is $d\left(\left(x_{1}, t_{1}\right),\left(x_{2}, t_{2}\right)\right)$ $=\sup \left(d\left(x_{1}, x_{2}\right),\left|t_{1}-t_{2}\right|\right)$. Any element of $H^{n}(X \times T)$ is equal to an element of $H^{n}(I \times J)$ where $I$ is a cover of $X$ and $J$ is a cover of $T$. Then $g_{t}=\left(f_{t}\right)_{I \times J}^{I}$ is defined by $g_{t}(U)=U \times V$ where $t \in V \in J$. If $|s-t|<$ $\delta(J)$, then $g_{s}$ and $g_{t}$ may be taken equal so, taking $\delta$ steps from 0 to 1 , we see that $g_{0}$ and $g_{1}$ induce the same map on $H^{n}(I \times J)$.

The metric complement of a totally bounded subspace consists of all points whose distance to the subspace is positive.

THEOREM 2.6. If $\left(X, X^{\prime}\right)$ is a totally bounded pair such that $H^{r}\left(X, X^{\prime}\right)$ $\neq 0$, then the metric complement of $X^{\prime}$ is nonempty.

Proof. Let $I$ be a cover of $X$ such that the map $H^{r}(I) \rightarrow H^{r}(X)$ is nonzero, and let $J$ be a cover of $X$ such that $\varepsilon(J)<\delta(I) / 2$. For each $V$ in $J$ choose $x_{V} \in V$. Either $d\left(x_{V}, X^{\prime}\right)>\delta / 4$ or $d\left(x_{V}, X^{\prime}\right)<\delta / 2$. If the former occurs for some $V$ in the finite family $J$, then we have constructed a point in the metric complement of $X^{\prime}$. If not, then for each $V$ in $J$ there is $y_{V}$ in $X^{\prime}$ such that $d\left(x_{V}, y_{V}\right)<\delta / 2$. Choose $U_{V} \in I$ such that $N_{\delta}\left(y_{V}\right) \subset$ $U_{V}$. Then $V \subset U_{V}$ and $U_{V} \in I^{\prime}$. Thus the map $H^{r}(I) \rightarrow H^{r}(J)$ is zero, contradicting the fact that the map $H^{r}(I) \rightarrow H^{r}(X)$ is nonzero.

3. Inverse limits of compact pairs. An inverse system of compact metric pairs is a family of compact metric pairs $X(i), i=1,2, \ldots$, and maps $f(i, j): X(j) \rightarrow X(i)$ defined for $i<j$ such that $f(i, k)=f(i, j) f(j, k)$ if $i<j<k$. To construct the inverse limit $X(\infty)$ of this system we first redefine the metric $d_{i}$ on $X(i)$ to be the equivalent metric $\inf \left(d_{i}, 1 / i\right)$ so that the diameter of $X(i)$ does not exceed $1 / i$. Define $X(\infty)$ to be 
$\left\{x \in \Pi X(i): x_{i}=f(i, j) x_{j}\right.$ for all $\left.i<j\right\}$, with $d(x, y)=\sup d_{i}\left(x_{i}, y_{l}\right)$. Define $f(i): X(\infty) \rightarrow X(i)$ to be projection on the $i$ th coordinate. In this section we shall prove the following theorem, after establishing two lemmas.

THEOREM 3.1. Let $\{X(i), f(i, j)\}$ be an inverse system of compact pairs with limit $\{X(\infty), f(i)\}$. If for each $i$ and $\delta>0$ there is $j>i$ so that $f(i) X(\infty)$ is $\delta$-dense in $f(i, j) X(j)$, then $H^{n}(X(\infty))$ is isomorphic to $\operatorname{Lim}_{\rightarrow} H^{n}(X(i))$.

A pair $\left(Y, Y^{\prime}\right)$ is $\delta$-dense in $\left(X, X^{\prime}\right)$ if $Y$ is $\delta$-dense in $X$ and $Y^{\prime}$ is $\delta$-dense in $X^{\prime}$. The $\delta$-density hypothesis always holds classically, but is necessary constructively as the following example shows.

EXAMPLE 3.2. Let $\left\{a_{i}\right\}$ be an increasing sequence of 0 's and 1's which cannot be all 0's. Let $X(i)$ be the closed unit disk if $a_{l}=0$, and the unit circle if $a_{i}=1$. Let $f(i, j)$ be the inclusion map $X(j) \subset X(i)$. Then $X(\infty)$ is the unit circle, so $H^{1}(X(\infty))$ is an infinite cyclic group. If Theorem 3.1 held in this case, then we could find a nonzero element of $\operatorname{Lim}_{\rightarrow} H^{1}(X(i))$, hence an $i$ such that $H^{1}(X(i)) \neq 0$. Thus we could find $i$ so that $a_{i}=1$. But this is Markov's principle.

Recall from [3] that an operation $g$ from a set $X$ to a set $Y$ assigns to each $x$ in $X$ an element $g(x)$ in $Y$. An operation need not be a function in that we may have $x_{1}=x_{2}$ without $g\left(x_{1}\right)=g\left(x_{2}\right)$.

LEMMA 3.3. Let I be a cover of $X(\infty)$ and $q$ a positive integer greater than $1 / \delta(I)$. If $\alpha>0$ is small enough, and $r>q$ is sufficiently large, then there is an operation $g: X(r) \rightarrow X(\infty)$ and a cover $R$ of $X(r)$ such that

(a) If $x, y \in X(\infty)$ and $d(f(q) x, f(q) y)<\alpha$, then $d(x, y)<\delta(I)$.

(b) If $x \in X(r)$, then $d(f(q) g x, f(q, r) x)<\alpha / 4$.

(c) If $x, y \in X(r)$ and $d(x, y)<\varepsilon(R)$, then $d(f(q, r) x, f(q, r) y)<$ $\alpha / 2$ and $d(g x, g y)<\delta(I)$.

Proof. Choose $\alpha>0$ so that if $u, v \in X(q)$ and $d(u, v)<\alpha$, then $d(f(i, q) u, f(i, q) v)<\delta(I)$ for all $i \leq q$. Then (a) holds. Choose $r$ large enough so that $f(q) X(\infty)$ is $\alpha / 4$ dense in $f(q, r) X(r)$. Then an operation $g$ satisfying (c) can be defined. Finally choose $R$ to satisfy the first inequality in (c). Then $d(f(q) g x, f(q) g y)<\alpha / 4+\alpha / 4+\alpha / 2$ so $d(g x, g y)<\delta(I)$ by $(\mathrm{a})$.

Note that (c) implies that $g_{I}^{R}$ is defined. We shall use the $g_{I}^{R}$ to construct an inverse to the map $\underset{\operatorname{Lim}}{\rightarrow} H^{n}(X(i)) \rightarrow H^{n}(X(\infty))$. First we show that the $g_{I}^{R}$ are compatible. 
LEMMA 3.4. Let II be a cover of $X(\infty)$, and I2 a refinement of I1. If $r_{3}$ is sufficiently large, and $R 3$ is a sufficiently fine cover of $X\left(r_{3}\right)$, then

$$
\left(g_{1}\right)_{I 1}^{R 1} f\left(r_{1}, r_{3}\right)_{R 2}^{R 3}=\mathrm{id}_{I 1}^{I 2}\left(g_{2}\right)_{I 2}^{R 2} f\left(r_{2}, r_{3}\right)_{R 2}^{R 3}
$$

Proof. Let $s=\max \left(r_{1}, r_{2}\right)$ and choose $\theta>0$ so that if $u, v \in X(s)$ and $d(u, v)<2 \theta$, then for $i=1,2$ we have

$$
d\left(f\left(r_{i}, s\right) u, f\left(r_{i}, s\right) v\right)<\varepsilon(R i) .
$$

Choose $r_{3}$ so $f(s) X(\infty)$ is $\theta$-dense in $f\left(s, r_{3}\right) X\left(r_{3}\right)$. Define $h: X\left(r_{3}\right) \rightarrow X(\infty)$ so that $d\left(f(s) h x, f\left(s, r_{3}\right) x\right)<\theta$ for all $x$ in $X\left(r_{3}\right)$. Let $R 3$ be a cover of $X\left(r_{3}\right)$ such that if $d(x, y)<\varepsilon(R 3)$, then $d\left(f\left(s, r_{3}\right) x, f\left(s, r_{3}\right) y\right)<\theta$. If $d(x, y)<\varepsilon(R 3)$, then $d\left(f(s) h x, f\left(s, r_{3}\right) y\right)<2 \theta$, so

$$
d\left(f\left(r_{i}\right) h x, f\left(r_{i}, r_{3}\right) y\right)<\varepsilon(R i) .
$$

Thus

$$
d\left(f\left(q_{i}\right) h x, f\left(q_{i}, r_{3}\right) y\right)<\alpha_{i} / 2
$$

so

$$
d\left(f\left(q_{i}\right) h x, f\left(q_{i}\right) g_{i} f\left(r_{i}, r_{3}\right) y\right)<\alpha_{i}
$$

whence

$$
d\left(h x, g_{i} f\left(r_{i}, r_{3}\right) y\right)<\delta(I i)
$$

Therefore

$$
h_{I 1}^{R 3}=\left(g_{1} f\left(r_{1}, r_{3}\right)\right)_{I 1}^{R 3}=\left(g_{1}\right)_{I 1}^{R 1} f\left(r_{1} r_{3}\right)_{R 1}^{R 3}
$$

and

$$
h_{I 2}^{R 3}=\left(g_{2}\right)_{I 2}^{R 2} f\left(r_{1}, r_{3}\right)_{R 2}^{R 3} .
$$

The theorem follows upon noting that $h_{I 1}^{R 3}=\mathrm{id}_{I 1}^{I 2} h_{I 2}^{R 3}$.

Proof of Theorem 3.1. Let $L=\operatorname{Lim}_{\rightarrow} H^{n}(X(i))$. The maps $f(i)^{*}$ : $H^{n}(X(i)) \rightarrow H^{n}(X(\infty))$ induce a unique map $\phi: L \rightarrow H^{n}(X(\infty))$. We will construct an inverse map $\psi$ to $\phi$, showing that $\phi$ is an isomorphism. If $I$ is a cover of $X(\infty)$, let $g$ and $R$ be as provided by Lemma 3.3. Then $\left(g_{I}^{R}\right)^{*}: H^{n}(I) \rightarrow H^{n}(R)$. Lemma 3.4 shows that these maps fit together to give a map $\psi$ from $H^{n}(X(\infty))$ to $L$. To show that $\phi \psi$ is the identity, let $I$ and $R$ be as above and $J$ a cover of $X(\infty)$ so that $f(r)_{R}^{J}$ is defined. If $x \in X(\infty)$, then

$$
d\left(f(q) x, f(q) g\left(x_{r}\right)\right)=d\left(f(q, r) x_{r}, f(q) g\left(x_{r}\right)\right)<\alpha / 4
$$


so $d\left(x, g\left(x_{r}\right)\right)<\delta(I)$, whence $\operatorname{id}_{I}^{J}=(g f(r))_{I}^{J}=g_{I}^{R} f(r)_{R}^{J}$. To show that $\psi \phi$ is the identity, let $I$ be a cover of $X(\infty)$ and $Q$ a cover of $X(q)$ where $q>1 / \delta(I)$ and $f(q)_{Q}^{I}$ is defined. Choose $\alpha<4 \delta(Q)$ and let $r, R$ and $g$ be as provided by Lemma 3.3. If $x \in X(r)$, then $d(f(q) g x, f(q, r) x)<\alpha / 4$ so id ${ }_{Q}^{R}=(f(q) g)_{Q}^{R}=f(q)_{Q}^{I} g_{I}^{R}$.

4. Polyhedral approximation. We need a systematic way to approximate compact subsets of a polyhedron, and their metric complements, by polyhedra. Let $K$ be a simplicial complex. A geometric realization $|K|$ of $K$ is obtained from a map $f$ taking the vertices of $K$ to Euclidean space in such a way that if $\left\{v_{0}, v_{1}, \ldots, v_{n}\right\}$ is an $n$-simplex of $K$, then $f\left(v_{0}\right), f\left(v_{1}\right), \ldots, f\left(v_{n}\right)$ are affine independent. The points of $|K|$ are sums $\sum a_{v} f(v)$ with $a_{v} \geq 0$ and $\sum a_{v}=1$ such that if $a_{v} \neq 0$ for every $v$ in some finite subset $J$, then $J$ is a simplex of $K$.

There is a natural map $H^{r}(K) \rightarrow H^{r}(|K|)$ defined as follows. Let $I$ be the cover of $|K|$ consisting of the sets star $v=\left\{\sum a_{u} f(u): a_{v} \neq 0\right\}$. Then $K$ and $I$ are isomorphic simplicial complexes under the correspondence $v \rightarrow \operatorname{star} v$. The desired map is the composite $H^{r}(K) \rightarrow H^{r}(I) \rightarrow H^{r}(|K|)$. This map is an isomorphism between $H^{r}(K)$ and $H^{r}(|K|)$ that is natural in the sense that it commutes with simplicial maps of $K$ and their induced continuous maps of $|K|$ (see [4; IX.9]).

Given a geometric realization $|K|$ of $K$ and a subcomplex $L$ of $K$, we get a geometric realization $|L|$ of $L$ in the obvious way. We get a geometric realization of the derived complex $K^{(1)}$ by mapping each point of $K^{(1)}$, which is a simplex of $K$, into the barycenter of its image in $|K|$. Clearly $|K|=\left|K^{(1)}\right|$ as point sets. If $L$ is a subcomplex of $K$, then we let $L$ denote the full subcomplex of $K^{(1)}$ whose vertices consist of those vertices of $K^{(1)}$ that are not in $L$.

THEOREM 4.1. Let $K$ be a simplicial complex and $|K|$ a geometric realization of $K$. Let $T$ be a totally bounded subspace of $|K|$. Then there is a sequence of subcomplexes $L_{l}$ of $K^{(i)}$ such that for each $i>0$

(a) $T \subset\left|L_{1}\right|$.

(b) Any subset of $|K|$ bounded away from $T$ is contained in $\left|\bar{L}_{l}\right|$ for some $i$.

(c) $\left|L_{1}\right| \supset\left|L_{2}\right| \supset \ldots$

Proof. We may assume that $|K|$ has diameter one. Choose $L_{l} \subset L_{l-1}^{(1)}$ inductively so that if $\sigma$ is a simplex in $L_{l}$, then $d(|\sigma|, T)<1 / 2 i$, while if $\sigma$ is a simplex of $K^{(i)}$ that is not in $L_{l}$, then $d(|\sigma|, T)>1 / 3 i$. Note that if $X$ is a subset of $|K|$ such that

$$
1 / 2 i+(d /(d+1))^{i}+(d /(d+1))^{i+1}<d(T, X)
$$

then $X \subset\left|\bar{L}_{l}\right|$. 
5. Alexander-Poincaré duality. Let $K$ be an abstract simplicial complex which is a combinatorial oriented $d$-manifold. Let $C A T$ be the set of pairs $(L, M)$ where $L$ is a subcomplex of the $i$ th derived complex $K^{(i)}$ of $K$ for some $i>0$, and $M$ is a subcomplex of $L$. Partially order $C A T$ by setting $\left(L_{1}, M_{1}\right) \leq\left(L_{2}, M_{2}\right)$ if $L_{1}^{(j)}$ is a subcomplex of $L_{2}^{(k)}$ and $M_{1}^{(j)}$ is a subcomplex of $M_{2}^{(k)}$ for some $j$ and $k$. We shall consider the partially ordered set $C A T$ to be a category in the usual way. Define a contravariant functor from $C A T$ to $C A T$ by assigning to each pair $(L, M)$ the pair $(\bar{M}, \bar{L})$, where $\bar{L}$ consists of those simplices of $K^{(i+1)}$ which have no vertex in common with $L^{(1)}$. Let $h: K^{(i+1)} \rightarrow K^{(l)}$ be a simplicial map taking a vertex of $K^{(i+1)}$, that is, a simplex of $K^{(i)}$, to one of its vertices. Let $\phi$ be the subdivision chain map. Define $H_{r}\left(L_{1}, M_{1}\right) \rightarrow H_{r}\left(L_{2}, M_{2}\right)$ and $H^{r}\left(L_{2}, M_{2}\right) \rightarrow H^{r}\left(L_{1}, M_{1}\right)$ by $h_{*}^{k} \phi^{j}$ and $\left(h_{*}^{k} \phi^{j}\right)^{*}$ respectively. As $h_{*} \phi$ and $\phi h_{*}$ are chain homotopic to the identity [4; VI.7.1], it is straightforward to verify that $H_{r}$ and $H^{r}$ are functors from $C A T$ to the category of finitely presented abelian groups.

THEOREM 5.1. There is a natural isomorphism

$$
H^{r}(L, M) \rightarrow H_{d-r}(\bar{M}, \bar{L}) .
$$

If $K$ is the $d$-skeleton of $a d+1$ simplex, then for $0 \leq r<d$ there is a natural isomorphism between the reduced groups

$$
\tilde{H}^{r}(L) \rightarrow \tilde{H}_{d-r-1}(\bar{L}) .
$$

Proof. Let $z$ be the $d$-chain that is the sum of the oriented $d$-simplices of $K$. Note that $z$ is a generator of $H_{d}(K)$. Let $\cap$ denote the cap product from $C^{r}(K) \otimes C_{d}(K)$ to $C_{d-r}(K)$. Define $D$ mapping $H^{r}(L, M)$ to $H_{d-r}(\bar{M}, \bar{L})$ by $D(x)=h^{*} x \cap \phi^{i+1} z$ where $L$ is a subcomplex of $K^{(i)}$. The proof that $D(x)$ is in $H_{d-r}(\bar{M}, \bar{L})$, and that $D$ is an isomorphism, may be found in $[5 ; 5.3 .13]$. The naturality of $D$ follows from the naturality of $\cap$ and the fact that $h_{*} \phi$ and $\phi h_{*}$ are chain homotopic to the identity. Specifically, one can verify that if $s$ is an $r$-cocycle in $K^{(t)}$, and $z$ is a $d$-cycle in $K^{(i)}$, then $\phi(s \cap z)=h^{*} s \cap \phi z$ and $h_{*}(s \cap z)=\phi^{*} s \cap h_{*} z$.

To prove the last assertion of the theorem consider the exact sequences

$$
\begin{array}{ccccccc}
H_{d-r}(K) & \rightarrow & H_{d-r}(K, \bar{L}) & \rightarrow & \tilde{H}_{d-r-1}(\bar{L}) & \rightarrow & \tilde{H}_{d-r-1}(K)=0 \\
H^{r}(K) & \rightarrow & H^{r}(L) & \rightarrow & \tilde{H}^{r}(L) & \rightarrow & 0 .
\end{array}
$$

By Theorem 5.1 the first two terms of the sequences are naturally isomorphic, whence so are the third terms. 
THEOREM 5.2. Let $K$ be the $d$-skeleton of $a d+1$ simplex, and $|K| a$ geometric realization of $K$. Let $T$ be a compact subspace of $|K|$. Then there is a sequence $P_{i}$ such that

(1) $P_{i}$ is a subcomplex of $K^{(i+1)}$,

(2) $P_{i}^{(1)}$ is a subcomplex of $P_{i+1}$,

(3) $\left|P_{i}\right|$ is bounded away from $T$,

(4) every subset of $|K|$ that is bounded away from $T$ is contained in some $\left|P_{i}\right|$,

(5) $H^{r}(T)=\lim _{\rightarrow} H_{d-r-1}\left(P_{i}\right)$.

Proof. Set $P_{i}=\bar{L}_{i}$ as constructed in Theorem 4.1. Part b of that theorem guarantees that $T$ is $\delta$-dense in $\left|L_{i}\right|$ if $i$ is sufficiently large, so $H^{r}(T)=\lim _{\rightarrow} H^{r}\left(\left|L_{i}\right|\right)$ by Theorem 3.1. Now $H^{r}\left(\left|L_{i}\right|\right)$ is naturally isomorphic to $H^{r}\left(L_{i}\right)$ because $H^{r}$ on compact pairs satisfies the EilenbergSteenrod axioms. Thus $H^{r}(T)=\lim _{\rightarrow} H^{r}\left(L_{i}\right)$. Finally, $H^{r}\left(L_{i}\right)$ is naturally isomorphic to $H_{d-r-1}\left(P_{i}\right)$ by Theorem 5.1.

6. The Jordan-Brouwer theorem. We are now ready to prove the Jordan-Brouwer theorem. First we state a few obvious facts about the subgroups of free groups that arise as zero-dimensional reduced cohomology groups.

LeMMa 6.1. For $V$ a set, let $G(V)$ denote the subgroup of the free group on $V$ whose coefficients sum to zero. Any function $f: V \rightarrow W$ induces $f_{*}: G(V) \rightarrow G(W)$, and $G(f(V))=f_{*}(G(V))$. If $f_{*}$ is one-to-one, then $f$ is one-to-one. If $V$ is a nonempty finite set, then the number of elements in $V$ is $1+\operatorname{rank} G(V)$.

THEOREM 6.2. Let $T$ be a totally bounded subset of the $d$-sphere with nonempty metric complement. If $H^{d-1}(T) \cong Z^{p}$, then the metric complement of $T$ can be written as $C^{0} \cup \cdots \cup C^{p}$ where $C^{k}$ is nonempty and polygonally connected away from $T$, and any path from $C^{k}$ to $C^{m}$ with $k \neq m$ comes arbitrarily close to $T$.

Proof. We may replace the $d$-sphere by a geometric realization $|K|$ of the $d$-skeleton $K$ of a $d+1$ simplex. Let $P_{1}, P_{2}, \ldots$ be as in Theorem 5.2. Taking $r=d-1$ in Theorem 5.2 we have $\lim _{\rightarrow} \tilde{H}_{0}\left(P_{i}\right) \cong Z^{p}$. By Theorem 1.2 we may reindex so that $\tilde{H}_{0}\left(P_{i}\right)$ is isomorphic to $A_{i} \oplus M_{i}$, where $M_{i}$ goes to zero in $\tilde{H}_{0}\left(P_{i+1}\right)$ and $A_{i}$ is mapped isomorphically onto $A_{i+1}$, so each $A_{i}$ is isomorphic to $Z^{p}$. Referring to Lemma 6.1 let $V$ be the set of components of $\left|P_{1}\right|$ and $W_{i}$ the set of components of $\left|P_{i}\right|$ for $i \geq 2$. Let $f(i): V \rightarrow W_{i}$ be induced by the inclusion $\left|P_{1}\right| \subset\left|P_{i}\right|$. Then $G(V)=$ $\tilde{H}_{0}\left(P_{1}\right)$ and $G\left(W_{i}\right)=\tilde{H}_{0}\left(P_{i}\right)$ and $f(i)_{*}$ is the map $\tilde{H}_{0}\left(P_{1}\right) \rightarrow \tilde{H}_{0}\left(P_{i}\right)$. By 
Lemma 6.1 we have $A_{i}=f(i)_{*} G(V)=G(f(i) V)$ and $\operatorname{card} f(i) V=1+$ $\operatorname{rank} G(f(i) V)=1+\operatorname{rank} A_{i}=1+p$. Moreover the map $f(i) V \rightarrow$ $f(i+1) V$ induced by the inclusion $\left|P_{l}\right| \subset\left|P_{l+1}\right|$ is one-to-one since $A_{i} \rightarrow$ $A_{i+1}$ is one-to-one. Thus we can write $f(i) V=\left\{C_{1}^{0}, \ldots, C_{1}^{P}\right\}$ where $C_{i}^{j} \subset$ $C_{i+1}^{j}$ and $\left|P_{i}\right| \subset C_{i+1}^{0} \cup \cdots \cup C_{i+1}^{p}$ and, if $j \neq k$, then $C_{i}^{j}$ is bounded away from $C_{i}^{k}$ by the minimum length of a one-simplex in $\left|K^{(i+2)}\right|$. Let $C^{j}=$ $\cup_{l \geq 1} C_{i}^{j}$ for $j=0, \ldots, p$.

Let $x \in|K|$ be such that $d(x, T)>0$. Choose $i$ so that $x \in\left|P_{i}\right|$. Then $x \in C_{i+1}^{j} \subset C^{j}$ for some $j$. If $x$ and $y$ are in $C^{j}$, then $x$ and $y$ are in $C_{i}^{j}$ for some $i$ and can be joined within $C_{i}^{j}$ by a polygonal path, which is necessarily bounded away from $T$. Finally, suppose $Q$ is a path from $C^{k}$ to $C^{m}$. Since $Q$ and $T$ are totally bounded, we can compute the distance $r$ from $Q$ to $T$. If $r>0$, then $Q \subset\left|P_{j}\right|$ for some $j$, so $Q$ joins $C_{j}^{k}$ to $C_{j}^{m}$, whence $k=m$. Thus if $k \neq m$, then $r=0$ so $Q$ comes arbitrarily close to $T$.

The sets $C^{i}$ need not be located. Let $T$ be the polygonal path $(0,0)$, $(1,1),(1, a),(2,0),(0,0)$ with $a \geq 0$. Then $T$ is a compact subset of $R^{2}$ and $H^{1}(T)=Z$. However if we could measure the distance from $(3,0)$ to that component of the complement of $T$ that contains $(1 / 2,1 / 4)$ then we could determine whether $a>0$.

A constructive theory of covering dimension for totally bounded spaces is developed in [7] and [2]. All we need here is that $\operatorname{Cov} X \leq n$ implies that there are arbitrarily fine covers $I$ of $X$ with no $n+1$ simplices.

COROLlaRY 6.3. Let $T$ be a totally bounded subset of the $d$-sphere. If $\operatorname{Cov} T \leq d-2$, then the metric complement of $T$ is nonempty and polygonally connected away from $T$.

Proof. As $\operatorname{Cov} T \leq d-2$, we can find arbitrarily fine covers of $T$ that have no $d-1$ or $d$ simplices. Hence $H^{d-1}(T)$ and $H^{d}(T)$ are zero. If $S^{d}$ is the $d$-sphere, then we have the exact sequence $H^{d}\left(S^{d}, T\right) \rightarrow H^{d}\left(S^{d}\right) \rightarrow$ $H^{d}(T)$, so $H^{d}\left(S^{d}, T\right) \neq 0$ whence, by Theorem 2.6 , the metric complement of $T$ in $S^{d}$ is nonempty. The corollary now follows from Theorem 6.2 with $p=0$.

To illustrate the connection between algebraic and metric information consider the polygonal path $T$ given by $(a, 1),(1,0),(0,0),(0,1),(a, 0)$ where $0 \leq a \leq 1 / 2$. From a classical point of view $T$ is either the triangle $(0,0)(0,1)(1,0)$, or is homotopic to the triangle $(0,0)(0,1)(a, 0)$, so $H^{1}(T)$ is infinite cyclic. If $H^{1}(T)$ were infinite cyclic from a constructive point of view, then the Jordan-Brouwer theorem would allow us to decide 
whether $(1 / 2,1 / 4)$ can be joined to $(0,2)$, and hence determine whether $a>0$. But this cannot be done for arbitrary $a$ in [0,1/2]. In fact $H^{1}(T)$ cannot be shown to be infinite cyclic from a constructive point of view. Indeed $H^{1}(T)$ is the direct limit of infinite cyclic groups where all the maps are isomorphisms with the possible exception that one map might be zero. If all the maps are isomorphisms, then we've got our hands on a generator. However if one of the maps down the line is zero, then our putative generator is zero, and the real generator appears beyond that point. As we have no way of determining which case we are in, we cannot get hold of a generator.

\section{REFERENCES}

1. G. Berg, W. Julian, R. Mines, and F. Richman, The constructive Jordan curve theorem, Rocky Mountain J. Math., 5 (1975), 225-236.

2. The constructive equivalence of covering and inductive dimensions, General Topology Appl., 7 (1977), 99-108.

3. E. Bishop, Foundations of Constructive Analysis, McGraw-Hill, 1967.

4. S. Eilenberg, and N. Steenrod, Foundations of Algebraic Topology, Princeton, 1952.

5. C. R. F. Maunder, Algebraic Topology, Van Nostrand, 1970.

6. F. Richman, A constructive modification of Vietoris homology, Fund. Math., 91 (1976), 231-240.

7. F. Richman, G. Berg, H. Cheng and R. Mines, Constructive dimension theory, Compositio Mathematica, 33 (1976), 161-177.

Received May 8, 1981 and in revised form February 17, 1982.

New Mexico State University

Las Cruces, NM 88003 



\section{PACIFIC JOURNAL OF MATHEMATICS EDITORS}

DONALD BABBITT (Managing Editor)

University of California

Los Angeles, CA 90024

Hugo Rossi

University of Utah

Salt Lake City, UT 84112

C. C. Moore and Arthur Ogus

University of California

Berkeley, CA 94720
J. DugunduI

Department of Mathematics

University of Southern California

Los Angeles, CA 90089-1113

R. FinN and H. SAmelson

Stanford University

Stanford, CA 94305

\section{ASSOCIATE EDITORS}
R. ARENS
E. F. BECKENBACH
B. H. NeUmaNN
F. WolF
K. YoshidA (1906-1982)

\section{SUPPORTING INSTITUTIONS}

UNIVERSITY OF ARIZONA

UNIVERSITY OF BRITISH COLUMBIA

CALIFORNIA INSTITUTE OF TECHNOLOGY

UNIVERSITY OF CALIFORNIA

MONTANA STATE UNIVERSITY

UNIVERSITY OF NEVADA, RENO

NEW MEXICO STATE UNIVERSITY

OREGON STATE UNIVERSITY
UNIVERSITY OF OREGON

UNIVERSITY OF SOUTHERN CALIFORNIA

STANFORD UNIVERSITY

UNIVERSITY OF HAWAII

UNIVERSITY OF TOKYO

UNIVERSITY OF UTAH

WASHINGTON STATE UNIVERSITY

UNIVERSITY OF WASHINGTON 


\section{Pacific Journal of Mathematics}

\section{Vol. 106, No. $1 \quad$ November, 1983}

John Ballard, Clifford's theorem for algebraic groups and Lie algebras . . . . . 1

Philip Richard Bartick, II and Edwin Duda, Finite-to-one open mappings

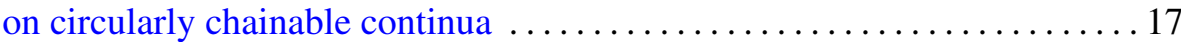

Frank Hayne Beatrous, Jr., $H^{\infty}$-interpolation from a subset of the

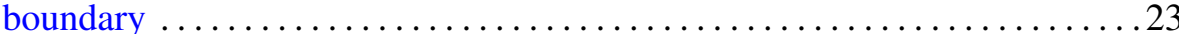

Sterling K. Berberian, Expectations in semifinite algebras ............ 33

Heron S. Collins and Wolfgang Ruess, Weak compactness in spaces of compact operators and of vector-valued functions $\ldots \ldots \ldots \ldots \ldots \ldots . \ldots 45$

David Downing and William O. Ray, Renorming and the theory of

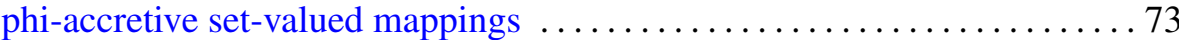

Harvey Charles Greenwald, On the theory of homogeneous Lipschitz

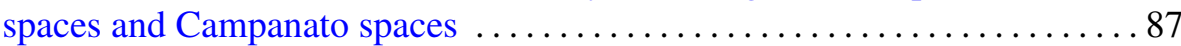

Irwen Valle Guadalupe and Lucio Ladislao Rodriguez, Normal curvature

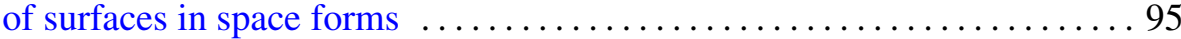

Boju Jiang, On the computations of the Nielsen number ............... 105

William H. Julian, Ray Mines, III and Fred Richman, Alexander

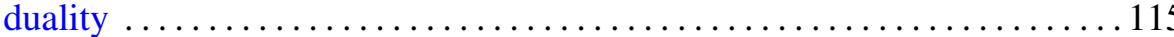

Midori Kobayashi, The connected component of the idèle class group of an

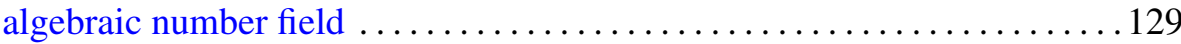

Ib Henning Madsen, Charles B. Thomas and C. Terence C. (Charles)

(Clegg) Wall, Topological spherical space form problem. III.

Dimensional bounds and smoothing

A. Sapounakis, The existence of strong liftings for totally ordered measure

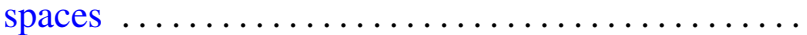

Gary Seitz, The root subgroups for maximal tori in finite groups of Lie type

Laurent Siebenmann and James M. Van Buskirk, Construction of irreducible homology 3-spheres with orientation reversing involution 